\title{
Utero-Fetal Unit and Pregnant Woman Modeling Using a Computer Graphics Approach for Dosimetry Studies
}

\author{
Jérémie Anquez, Tamy Boubekeur, Lazar Bibin, Elsa Angelini, \\ and Isabelle Bloch
}

Telecom ParisTech, CNRS UMR 5141 LTCI, Paris, France

\begin{abstract}
Potential sanitary effects related to electromagnetic fields exposure raise public concerns, especially for fetuses during pregnancy. Human fetus exposure can only be assessed through simulated dosimetry studies, performed on anthropomorphic models of pregnant women. In this paper, we propose a new methodology to generate a set of detailed utero-fetal unit (UFU) 3D models during the first and third trimesters of pregnancy, based on segmented 3D ultrasound and MRI data. UFU models are built using recent geometry processing methods derived from mesh-based computer graphics techniques and embedded in a synthetic woman body. Nine pregnant woman models have been generated using this approach and validated by obstetricians, for anatomical accuracy and representativeness.
\end{abstract}

\section{Introduction}

Several organizations and institutes like the World Health Organization or the Mobile Manufacturers Forum, have pointed out the need for studies of interactions between electromagnetic fields and biological tissues. For this purpose, experiments performed in vivo on animals and in vitro at the cellular level are complemented by simulated dosimetry studies. These studies require rasterized computational body models in order to simulate the electromagnetic field and derive the energy absorption at each point of the model.

With the advent of fast whole body acquisition imaging protocols, voxel-based models are nowadays built using segmented medical data acquired on volunteers. Numerous adult and children voxel-based models are available [1], which have enabled extensive dosimetric studies. In 2006, the World Health Organization designated studies aiming at assessing fetal exposure during pregnancy as a new priority. Since gathering whole body medical data on a pregnant woman is unethical, hybrid models were built by merging stylized models with organs built with surface equations, voxel-based models and/or synthetic models from the computer graphics community. Only few works modeled pregnant women at different stages of pregnancy. However, detail and realism of the UFU models remained limited in these models, and did not allow precise fetal exposure assessment. Stylized models were used in 2, which are inherently simplified anatomical representations. 
A detailed set of models of pregnant women was already proposed in [3], but was limited by the fact that the fetus models contained a realistic but coarse skeleton extracted from a CT scan, scaled and combined with different synthetic envelopes not generated from medical data. Moreover, only a limited set of fetal tissues were distinguished (including soft tissues, skeleton, brain). The set of models proposed in 4 was the only one built using medical images, but UFU models at different gestational ages were obtained by scaling a single model extracted from MRI data obtained at 35 weeks of amenorrhea (WA). This transformation seems inappropriate as fetal limbs and organs do not develop at the same moment nor grow linearly. Therefore, there is still a need for more complex models.

In this paper, we propose to fill this gap by building a set of pregnant woman models, embedding detailed and realistic UFU models. Realism is ensured by the use of medical images, obtained with two modalities used in routine pregnancy follow up: 3D ultrasound (3DUS) during the first trimester and magnetic resonance imaging (MRI) during the second and third trimesters. Detailed segmentations, validated by expert physicians, are then processed using several point-based graphics tools, in particular the MLS operator, which have recently emerged as efficient and robust techniques in digital geometry processing. We introduce their use in medical imaging by meshing smooth surfaces sampled from medical volume data. This mesh representation preserves smoothness during the discretization of the UFU anatomy on the Cartesian grid used for dosimetric simulations, which is crucial as surface singularities induce bias in the simulation results. UFU models are merged with a synthetic woman model, overlapping the maternal bulk extracted from the images and under control of obstetricians.

The originality of this work is three-fold: an emerging problematic is addressed; an extensive database of images acquired with state-of-the-art modalities used in obstetrics has been gathered (Section 2); recent geometry processing methods derived from mesh-based computer graphics techniques are used to provide a smooth representation of the UFU anatomical structures (Section 3). Preliminary results and future works are presented in Section 4 .

\section{Images Database and Utero-Fetal Unit Segmentation}

3DUS Image Data. With the collaboration of obstetricians from the PortRoyal and Beaujon hospitals (Paris, France), we obtained eighteen 3DUS images between 8 and 14 WA. These images have a submillimetric and isotropic resolution (typically $0.6 \times 0.6 \times 0.6 \mathrm{~mm}^{3}$ ) and contain the whole UFU. First, voxels are classified into two classes, the amniotic fluid on the one hand, and the fetal and maternal tissues on the other hand. This is performed using the method described in [5], where statistical distributions of voxel intensities within each class are exploited in a deformable model segmentation framework. Then, the fetus is manually disconnected from the uterine wall and from the umbilical cord. Finally, the placenta and the endometrium are identified.

MRI Image Data. In collaboration with pediatric radiologists from the CochinSt Vincent de Paul hospital (Paris, France), a database of twenty-two routine 


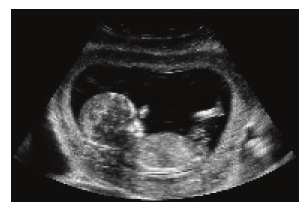

(a)

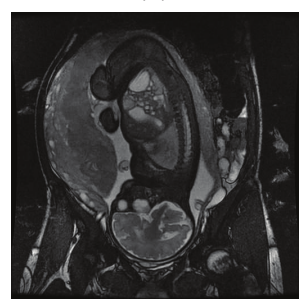

(c)

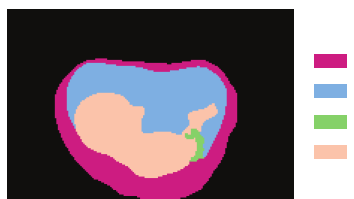

(b)

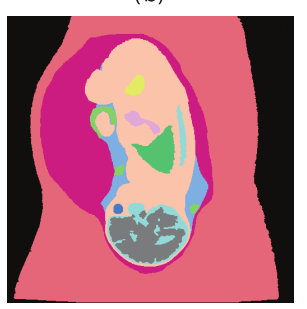

(d)

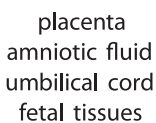

maternal tissues

placenta/uterine wall amniotic fluid umbilical cord brain cerebrospinal fluid lungs stomach urinary bladder eyes other fetal tissues

Fig. 1. 3DUS (a) and MRI (c) images at 13 and 34 WA, with corresponding segmentation results (b) and (d)

MRI exams has been gathered, of fetuses between 26 and 35 WA. Images were acquired using the Balanced Steady State Free Precession sequence, which enables to acquire volumes encompassing the UFU in less than thirty seconds. Thus, images free from fetal motion related artifacts can be obtained, guaranteeing three dimensional consistency of the UFU anatomical structures. Moreover, intensities in fluids are strikingly higher than in fetal soft tissues and high contrasts enable easy delineation of the fetus and its anatomy [6]. The typical images resolution is $1 \times 1 \times 4 \mathrm{~mm}^{3}$. First, maternal tissues, uterine wall, placenta, amniotic fluid, umbilical cord and fetus are segmented using semi-automatic segmentation tools. Then, fetal anatomy is refined by segmenting the brain, cerebrospinal fluid, spine, eyes, lungs, heart, stomach and urinary bladder. The overall segmentation is time consuming and automated approaches are currently being developed. Accurate results have already been obtained for eyes, brain [7, cerebrospinal fluid and spine automated segmentation. Typical 3DUS and MRI data images are shown in Figure 1, together with the corresponding segmentation results, which were individually validated by obstetricians.

\section{Utero-Fetal Unit and Pregnant Woman Modeling}

\subsection{Surface Reconstruction}

For dosimetry studies, Finite Difference Time Domain method is frequently used for numerical simulations on a spatial grid, with labeled anthropomorphic models which need to be smooth to avoid simulation bias on singularities. Naive direct meshing approaches generate "steps" effects (see Figure 2) in the surface models, especially when MRI images with anisotropic resolution are considered. In this section, we describe a method for generating a high quality triangle mesh from a presegmented volume of interest (see Figure 2). We adopt a generic approach 


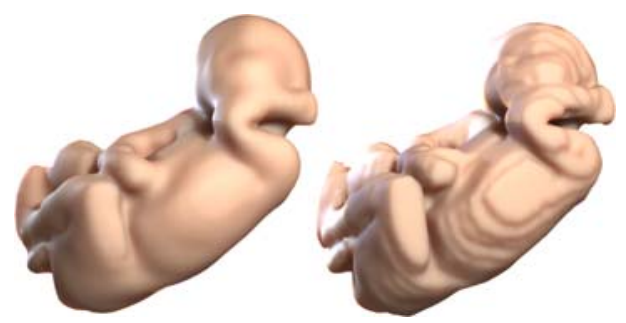

Fig. 2. Illustration on a fetus body of the proposed surface reconstruction (left) versus direct meshing from image segmentation (right)

by first extracting an unorganized set of points from the volume data and then generating a mesh by sampling a smooth surface which approximates this point set. The entire process is performed in a few seconds.

The mesh reconstruction algorithm is composed of three main steps, detailed below (see Figure 3 for an illustration). With this algorithm, a mesh of arbitrary resolution can be extracted from an arbitrary sparse point set. Consequently, the system allows producing dense enough surface sampling to enable both accurate subsequent deformations of the objects and robust rasterization within the original volume domain. Point-Normal Sampling. In addition to the position $\mathbf{p}_{i}$ of a point sample, the algorithm requires a surface normal estimate $\mathbf{n}_{i}$ at this point, in order to generate a mesh $\mathcal{M}$ from the point-normal sampling $\mathcal{P}^{N}=\left\{\left\{\mathbf{p}_{0}, \mathbf{n}_{0}\right\}, \ldots\left\{\mathbf{p}_{m}, \mathbf{n}_{m}\right\}\right\}$. We compute an approximate normal vector at each point by using a Principal Component Analysis [8] in 3D space. More precisely, the normal is given as $\mathbf{u}_{i}$, the eigen vector associated with the smallest eigen value of the covariance matrix of the $k$-nearest-neighborhood of $\mathbf{p}_{i}$. In practice, the anisotropic nature of the original sampling induces a rather large value for $k$, usually $k \in[50,120]$, and we use a $\mathrm{kD}$-Tree data structure [9] to efficiently compute the $k$-neighborhood queries.

In our case, the sign of the normal can be resolved using additional information available from the volume data: the vector $\mathbf{a}_{i}$, from the surface point $\mathbf{p}_{i}$ to the corresponding point on a dilated volume boundary, always points from the surface to the outside of the object. Thus, we get

$$
\mathbf{n}_{i}=\frac{\overline{\mathbf{n}_{i}}}{\left\|\overline{\mathbf{n}_{i}}\right\|} \text { with } \overline{\mathbf{n}_{i}}=\left\{\begin{array}{ll}
\mathbf{u}_{i} & \text { if } \mathbf{u}_{i} \cdot \mathbf{a}_{i}>0 \\
-\mathbf{u}_{i} \text { otherwise }
\end{array} .\right.
$$

Note that $\mathbf{n}_{i}$ is only an estimate, with a smoothness controlled by $k$. To increase the quality of this estimate for later stages of the reconstruction pipeline, the normals are re-estimated after each major processing step in this algorithm.

MLS Operator. The Moving Least Square (or MLS) projection operator 1011] is a scattered data approximation method intensively used in geometry processing. We make use of it for both filtering and meshing steps, as it provides a simple and powerful tool for the sparse sampling $\mathcal{P}^{N}$ extracted from volume data. We 


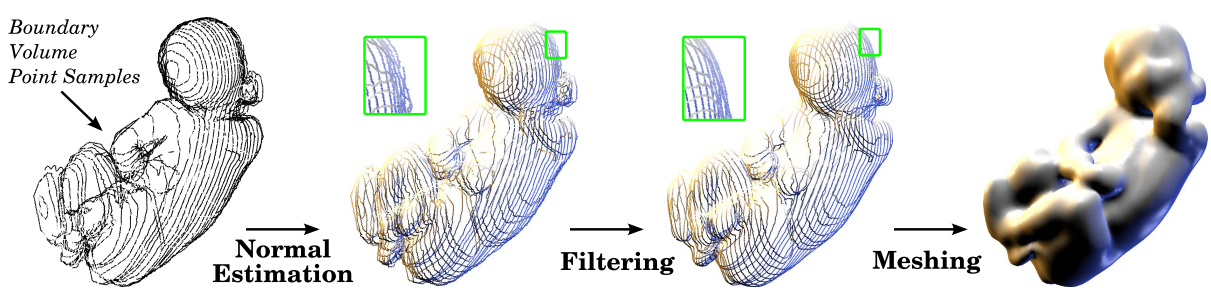

Fig. 3. Illustration on a fetus body of the reconstruction pipeline

consider a variation of a recent simplification of the MLS operator [12. Let consider $\mathbf{q} \in \mathbb{R}^{3}$. The MLS operator is defined as:

$$
M L S^{\mathcal{P}^{N}}: \mathbb{R}^{3} \rightarrow \mathbb{R}^{3}, \mathbf{q} \rightarrow \Pi^{\infty}(\mathbf{q})
$$

It is based on the orthogonal projection $\Pi(\mathbf{q})$ of $\mathbf{q}$ onto a weighted least square plane $H_{\mathbf{q}}=\{c(\mathbf{q}), n(\mathbf{q})\}: \Pi(\mathbf{q})=\mathbf{q}-<\mathbf{q}-c(\mathbf{q}) \cdot n(\mathbf{q})>n(\mathbf{q})$ where

$$
c(\mathbf{q})=\frac{\sum_{i} \omega\left(\left\|\mathbf{q}-\mathbf{p}_{\mathbf{i}}\right\|\right) \mathbf{p}_{\mathbf{i}}}{\sum_{i} \omega\left(\left\|\mathbf{q}-\mathbf{p}_{\mathbf{i}}\right\|\right)} \text { and } n(\mathbf{q})=\frac{\sum_{i} \omega\left(\left\|\mathbf{q}-\mathbf{p}_{\mathbf{i}}\right\|\right) \mathbf{n}_{\mathbf{i}}}{\left\|\sum_{i} \omega\left(\left\|\mathbf{q}-\mathbf{p}_{\mathbf{i}}\right\|\right) \mathbf{n}_{\mathbf{i}}\right\|}
$$

define a weighted combination over a local neighborhood of surface point samples near $\mathbf{q}$ with $\left\{\mathbf{p}_{i}, \mathbf{n}_{i}\right\} \in \mathcal{P}^{N}$. For efficiency reasons, we use Wendland's [13] compactly supported, piecewise polynomial function as the weighting kernel:

$$
\omega(t)=\left\{\begin{array}{ll}
\left(1-\frac{t}{h}\right)^{4}\left(\frac{4 t}{h}+1\right) & \text { if } 0 \leq t \leq h \\
0 & \text { if } t>h
\end{array},\right.
$$

where $h$ controls the size of the support (i.e. smoothness). By applying iteratively this projection procedure, we define $\Pi^{i+1}(\mathbf{q})=\Pi\left(\Pi^{i}(\mathbf{q})\right)$ and generate a sequence of points $\left\{\mathbf{q}, \Pi(\mathbf{q}), \ldots, \Pi^{i}(\mathbf{q}), \ldots\right\}$ which - considering $\mathbf{q}$ in the vicinity of $\mathcal{P}^{N}[14]$ - converges toward a stationary point $\Pi^{\infty}(\mathbf{q})$. The set of points in $\mathbb{R}^{3}$ which are stationary by this MLS projection of $\mathcal{P}^{N}$ is called the point set surface (or PSS) [15] of $\mathcal{P}^{N}$. This procedure converges very quickly in the vicinity of $\mathcal{P}^{N}$. In practice, we bound the number of iterations to 5 and the precision to a user-defined value, i.e. $\left\|\Pi^{i+1}(\mathbf{q})-\Pi^{i}(\mathbf{q})\right\|<\epsilon$. Note that $\omega(t)$ has a compact support, which allows us to consider only a small and local set of neighbors in $\mathcal{P}^{N}$. Again, a kD-Tree is used to query them in logarithmic time. Filtering. The manual segmentation may often lead to inaccurate volume boundary and unwanted samples in $\mathcal{P}^{\mathcal{N}}$. Consequently, we need to filter $\mathcal{P}^{\mathcal{N}}$ prior to the mesh generation stage. In practice, this filtering boils down to smooth $\mathcal{P}^{\mathcal{N}}$ and remove its outliers, which are samples located far away from the estimated surface. The former can be addressed by applying the MLS projection on every sample of $\mathcal{P}^{\mathcal{N}}$, using $h$ to control the low-pass filtering effect (i.e. $\mathcal{P}^{\mathcal{N}}$ is projected onto the PSS it defines). We address the later problem using an iterative classification inspired from the method of Bradley et al. [16]: we compute the Plane Fit Criterion proposed by Weyrich et al. [17, remove the detected outliers and restart with a 


\section{(1)}

Fig. 4. Pregnant woman modeling: original trunk of the synthetic woman (left), deformed trunk (center) and final model (right)

quadratically decreasing bound until a user-defined threshold. Our experiments show that the number of iterations can be fixed to 3. Mesh Generation. We finally generate the mesh $\mathcal{M}$ from $\mathcal{P}^{N}$ using two distinct approaches:

- in most cases, we use the implicit form of the PSS:

$$
f^{\mathcal{P}^{N}}: \mathbb{R}^{3} \rightarrow \mathbb{R}, \mathbf{q} \rightarrow n(\mathbf{q})^{\top}(\mathbf{q}-c(\mathbf{q}))
$$

to define a scalar field with zero set $-f^{\mathcal{P}^{N}}(\mathbf{q})=0-$ corresponding to a smooth surface approximating $\mathcal{P}^{N}$. We contour it by feeding the extended marching cube algorithm [18] with $f^{\mathcal{P}^{N}}$ (i.e., $f^{\mathcal{P}^{N}}$ is evaluated at marching cube grid vertices).

- in some cases, exhibiting large missing surface regions in the sampling obtained from volume data, we use the Poisson Reconstruction algorithm, as proposed by Kazhdan et al. [19]. We use their code, publicly available.

As a result, we obtain a triangle mesh $\mathcal{M}$ sampling at arbitrary precision (controlled by the marching cube grid size) a smooth surface defined from the input boundary samples extracted from volume data. Surface meshes for all segmented objects in Figure 1 have been generated with our method. These mesh models can then be used for further interactive skinning, deformation and visualization.

\subsection{Pregnant Woman Modeling}

No segmented woman model is freely available and an approach similar to 4 . has been adopted. Pregnant women models are built by inserting UFU models into a synthetic woman model, Victoria, distributed by DAZ studio (www.daz3d.com). UFU models generated using ultrasound data are registered rigidly into Victoria. Those models correspond to early pregnancy stages. Consequently, Victoria's body is not altered, as the abdominal morphology of a pregnant woman is not modified at such stages by the growing UFU, given its small volume.

However, a different insertion process has to be considered when models generated from MRI data are inserted (see Figure 4). First, UFU and the maternal bulk models are positioned by rigidly registering the pubis. Then, a realistic inclination is defined. Finally, Victoria's thigh and trunk are morphed using free form deformations to fit the maternal bulk. The final pregnant woman model is obtained by removing the maternal bulk. Positioning and morphing operations were supervised by experienced obstetricians. 

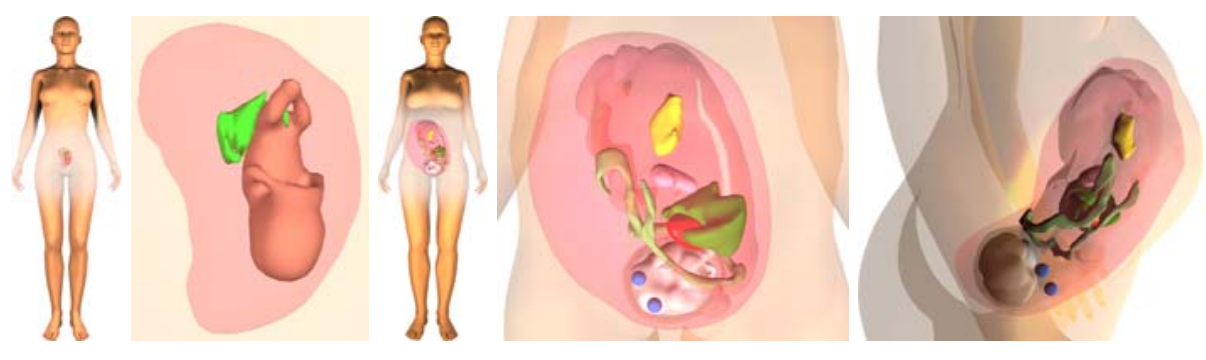

Fig. 5. Pregnant women models at 13 WA (left two images) and 35 WA (right three images), generated using US3D and MRI data, respectively

\section{Preliminary Results and Future Works}

Nine pregnant woman models have been generated using the method presented in this paper: four from 3DUS images (8, 9, 10 and $13 \mathrm{WA})$ and five from MRI images $(30,32,32,33$ and $35 \mathrm{WA})$. Models at 13 and $35 \mathrm{WA}$ are shown in Figure 5. All models were anatomically validated by obstetricians and pediatric physicians, and will be shared with other researchers in a near future. Preliminary dosimetry studies on models derived from MRI have been performed and results show that fetal position and morphology have a direct influence on fetal exposure [20], demonstrating the importance of considering multiple models with similar gestational ages.

Future works include the computation of additional models, especially for the second pregnancy trimester. During this period, 3DUS cannot be used due to limited field of view and MRI images quality is often altered by fetal motion related artifacts. However, an artifact free MRI volume was recently obtained at $26 \mathrm{WA}$ and the corresponding model is currently under construction. A fetus model has also been articulated, to be morphed into different anatomical position. This enables the study of the posing influence on dosimetry. Finally, this model can be used as a tool for segmentation. Indeed, registering this model on fetal landmark points (e.g. joints cartilage) in a new image dataset to initialize a graphcut segmentation provided promising results in preliminary experiments for the fetal envelope segmentation.

Acknowledgments. This work has been partially founded by a grant from the Fondation Santé et Radiofréquences and by Orange Labs. The authors thank all the radiologists and obstetricians involved in the models building process.

\section{References}

1. Caon, M.: Voxel-based computational models of real human anatomy: a review. Radiation and Environmental Biophysics 42(4), 229-235 (2004)

2. Dimbylow, P.: Development of pregnant female, hybrid voxel-mathematical models and their application to the dosimetry of applied magnetic and electric fields at 50 Hz. Physics in Medicine and Biology 51(10), 2383-2394 (2006) 
3. Xu, X., Taranenko, V., Zhang, J., Shi, C.: A boundary-representation method for designing whole-body radiation dosimetry models: pregnant females at the ends of three gestational periods-RPI-P3,-P6 and-P9. Physics in Medicine and Biology 52(23), 7023-7044 (2007)

4. Wu, D., Shamsi, S., Chen, J., Kainz, W.: Evaluations of specific absorption rate and temperature increase within pregnant female models in magnetic resonance imaging birdcage coils. IEEE Transactions on Microwave Theory and Techniques 54(12 Part 2), 4472-4478 (2006)

5. Anquez, J., Angelini, E., Bloch, I.: Segmentation of fetal 3D ultrasound based on statistical prior and deformable model. In: ISBI, pp. 17-20 (2008)

6. Anquez, J., Angelini, E., Bloch, I., Merzoug, V., Bellaiche-Millischer, A., Adamsbaum, C.: Interest of the Steady State Free Precession (SSFP) sequence for 3D modeling of the whole fetus. In: EMBS, pp. 771-774 (2007)

7. Anquez, J., Angelini, E., Bloch, I.: Automatic segmentation of head structures on fetal MRI. In: ISBI (2009)

8. Hoppe, H., DeRose, T., Duchamp, T., McDonald, J., Stuetzle, W.: Surface reconstruction from unorganized points. In: ACM SIGGRAPH (1995)

9. Bentley, J.L.: Multidimensional binary search trees used for associative searching. Communications of the ACM 18(9), 509-517 (1975)

10. Levin, D.: The approximation power of moving least-squares. Mathematics of Computation $67(224), 1517-1531$ (1998)

11. Levin, D.: Mesh-independent surface interpolation. Geometric Modeling for Scientific Visualization 3 (2003)

12. Alexa, M., Gross, M., Pauly, M., Pfister, H., Stamminger, M., Zwicker, M.: Pointbased computer graphics. In: ACM SIGGRAPH Course Notes (2004)

13. Wendland, H.: Piecewise polynomial, positive definite and compactly supported radial functions of minimal degree. Advances in Computational Mathematics 4(4), 389-396 (1995)

14. Amenta, N., Kil, Y.J.: Defining point-set surfaces. In: ACM SIGGRAPH, pp. 264270 (2004)

15. Alexa, M., Behr, J., Cohen-Or, D., Fleishman, S., Levin, D., Silva, C.T.: Point set surfaces. In: Vis, pp. 21-29 (2001)

16. Bradley, D., Boubekeur, T., Heidrich, W.: Accurate multi-view reconstruction using robust binocular stereo and surface meshing. In: CVPR, pp. 1-8 (2008)

17. Weyrich, T., Pauly, M., Keiser, R., Heinzle, S., Scandella, S., Gross, M.: Postprocessing of scanned 3d surface data. In: PBG, pp. 85-94 (2004)

18. Kobbelt, L., Botsch, M., Schwanecke, U., Seidel, H.P.: Feature sensitive surface extraction from volume data. In: ACM SIGGRAPH, pp. 57-66 (2001)

19. Kazhdan, M., Bolitho, M., Hoppe, H.: Poisson surface reconstruction. In: SGP, pp. 61-70 (2006)

20. Bibin, L., Anquez, J., Hadjem, A., Angelini, E.D., Wiart, J., Bloch, I.: Dosimetry studies on a fetus model combining medical image information and synthetic woman body. In: WC (2009) 